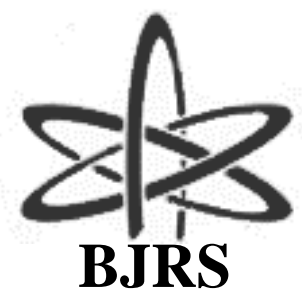
BRAZILIAN JOURNAL
$\mathrm{OF}$
RADIATION SCIENCES
09-02B (2021) 01-11

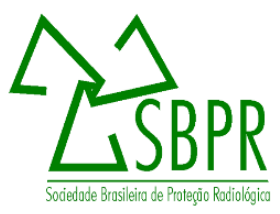

\title{
Knowledge base about risk and safety of nuclear facilities to support analysts and decision-makers
}

\author{
Antonio Souza Vieira Neto, Amaury Muñoz Oliva, Patrícia da Silva Pagetti de \\ Oliveira, Marcos Costa Hunold, Maria Eugênia Lago Jacques Sauer, Vinicius Andrea \\ Instituto de Pesquisas Energéticas e Nucleares (IPEN - CNEN/SP) \\ asvneto@ipen.br
}

\begin{abstract}
Epistemic uncertainty (related to lack of knowledge), often found in the documentation of nuclear facility engineering projects, can affect the decision-making process of managers and analysts on safety and risk issues. This article conceptualizes the nature of the major uncertainties involved in engineering projects. It describes a knowledge base developed to gather data and information related to an Open-Pool Light-water Research Reactor (OPLRR) project and whose purpose is to assist professionals who work in nuclear facilities. To reduce the epistemic uncertainties that may arise in the project, the OPLRR knowledge base is designed with a set of information that allows identifying and facilitating the forwarding of solutions to address inconsistencies and/or pending issues that may exist in the project. In this sense, the information and the documents related to the project are organized in a graphical and hierarchical architecture, allowing the knowledge base users to quickly and easily obtain information regarding the systems, processes, equipment, and components of the project. Besides that, a set of documents containing descriptions, reliability data, and some other information about the systems and components are specially created to the knowledge base, and it is crucial to reduce epistemic uncertainties once it raises the issues and the inconsistencies of the project, as well as it clarifies the interrelations between the systems, the functioning of the equipment, their failures modes and the consequences of their failures, and some other data, which are not originally contained in the documents of the project.
\end{abstract}

Keywords: knowledge base, epistemic uncertainty, research reactor. 


\section{INTRODUCTION}

A major challenge in project management is dealing with uncertainties that give rise to outcomes that are unknown or only imprecisely known [1]. As the Chinese philosopher, Lao Tsu stated, "Knowing ignorance is strength. Ignoring knowledge is sickness" [2].

Despite the relevance of the challenge mentioned above, during centuries the concepts of uncertainty and risk were comprehended as having similar meanings. Only at the beginning of the XX century, the concept of uncertainty has been better understood, when Frank H. Knight emphasized the conceptual difference between risk and uncertainty. According to him, the risk is considered as a measurable probability, and uncertainty as a situation expressed by indeterminate and non-quantifiable values, that is, it refers to a situation of "numerically immeasurable" [3]. Since Knight's definition, an extensive body of knowledge - theoretical as well as technical - related to uncertainty has already been created by scientists and practitioners of all disciplines. However, there is no agreement on the best taxonomy to be used. Despite this, when examining the nature of uncertainty, two categories have been accepted in the technical literature, ontological uncertainty and epistemic uncertainty $[4,5]$.

Ontological uncertainty is due to the inherent variability of the system and represents a state of complete ignorance. Not only do we not know, but we do not even know what we do not know, therefore nothing can be done to reduce this uncertainty. In a technical context, it includes technological surprises or unexpected consequences caused by new developments or breakthroughs in technology and/or infrastructure [5]. In a social context, ontological uncertainty includes value and belief diversities arisen from cultural, social, economic, and political dynamics [5].

Epistemic uncertainty (sometimes referred to as subjective) is due to imperfect or incomplete knowledge of the system of interest. If we have the proper information, this uncertainty can be reduced. This is the fundamental difference between ontological and epistemic uncertainty [5], and this was the main reason why we proposed in this paper the development of a knowledge base designed to reduce the epistemic uncertainties involving decision-making by managers and analysts on issues involving the safety of nuclear research facilities in the design phase. 
Regarding decision-making processes in large scale projects, one of the most significant challenges is to deal with the uncertainties that may arise from incomplete information, disagreements between different sources of information, linguistic imprecision, inaccuracy or scarcity of numerical data, inadequacy or limitation of calculation methods, among others. These uncertainties are characterized as epistemic and can be more clearly identified and diminished if a better way of collecting and transmitting the data is implemented.

To reduce these epistemic uncertainties in engineering project development, a comprehensive knowledge base has been proposed. This knowledge base incorporates information that describes the project objectively and analyzes it from a variety of perspectives. Information about ambiguities, inconsistencies, and lack of information related to project documentation is also included in the knowledge base. The purpose of this knowledge base is to assist the decisionmaking processes of managers, supervisors, engineers, operators, and other professionals working in the safety, design, operation, and maintenance of nuclear facilities.

\section{STRUCTURE OF THE KNOWLEDGE BASE}

This section describes the knowledge base being developed to support the design development of an Open Water Pools Research Reactor (OPLRR) project.

This knowledge base is structured to contain a set of information that allows identifying and facilitating the forwarding of solutions to address inconsistencies and/or outstanding issues that may exist in the project. For that reason, the documentation of the OPLRR, such as P\&I diagram, interfaces between the systems, setpoints, drawings, descriptive memorials, and even inconsistencies or ambiguities found among the documents, is organized and presented in interconnection diagrams, with a structure similar to those found in concept maps.

These interconnection diagrams have been built using the CmapTools, a free software originally designed to help in knowledge management [6].

The use of this kind of tool allows the information to be organized in such a manner that the user can search, visualize and obtain it in a more objective and clearer dataset, reducing the 
epistemic uncertainty through the search and making it possible to create a solid knowledge base to the users.

The information has been structured as follows: the systems and the reactor structures, such as the core cooling or shutdown systems are presented in form of visual blocks similar to those commonly used to represent the concepts within a concept map structure.

The user can verify the information about that specific system by clicking or passing the mouse over the visual block representing it, once all the relevant documents related to that system are attached to its visual block and displayed as icons.

Lines connecting the visual blocks indicate what are the interfaces or interactions existing between those items, as exemplified in Figure 1, which demonstrates an example of the interconnection diagram of a typical OPLRR First Shutdown System (FSS) and its interactions or dependencies with other systems, such as the electrical or the pneumatic ones.

The main system is presented at the center and in a blue visual block. The other systems that somehow have an interconnection with the main system are presented in an orange visual block.

Figure 1. Interconnection diagram representing the First Shutdown System (FSS) of the reactor.

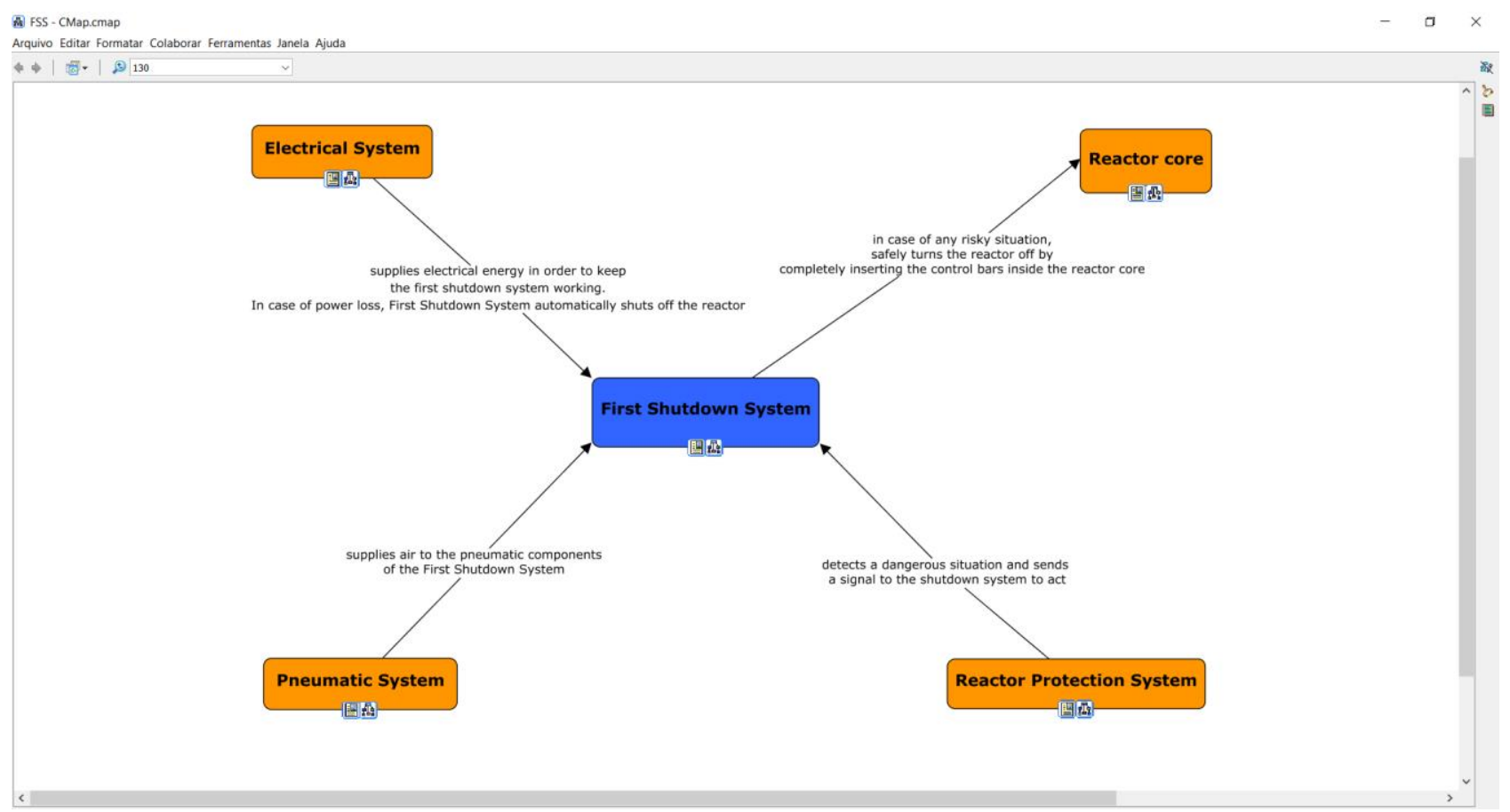


Subsystems or a more detailed description of a given system are shown in another interconnection diagram that can be easily accessed by a link exhibited at its visual root block.

In Figure 2 it is shown the reactor core's interconnection diagram popping up when the user clicks on its visual block located on the FSS interconnection diagram.

Figure 2. Representation of reactor core interconnection diagram popping up from the FSS interconnection diagram when the user clicks over its visual block.

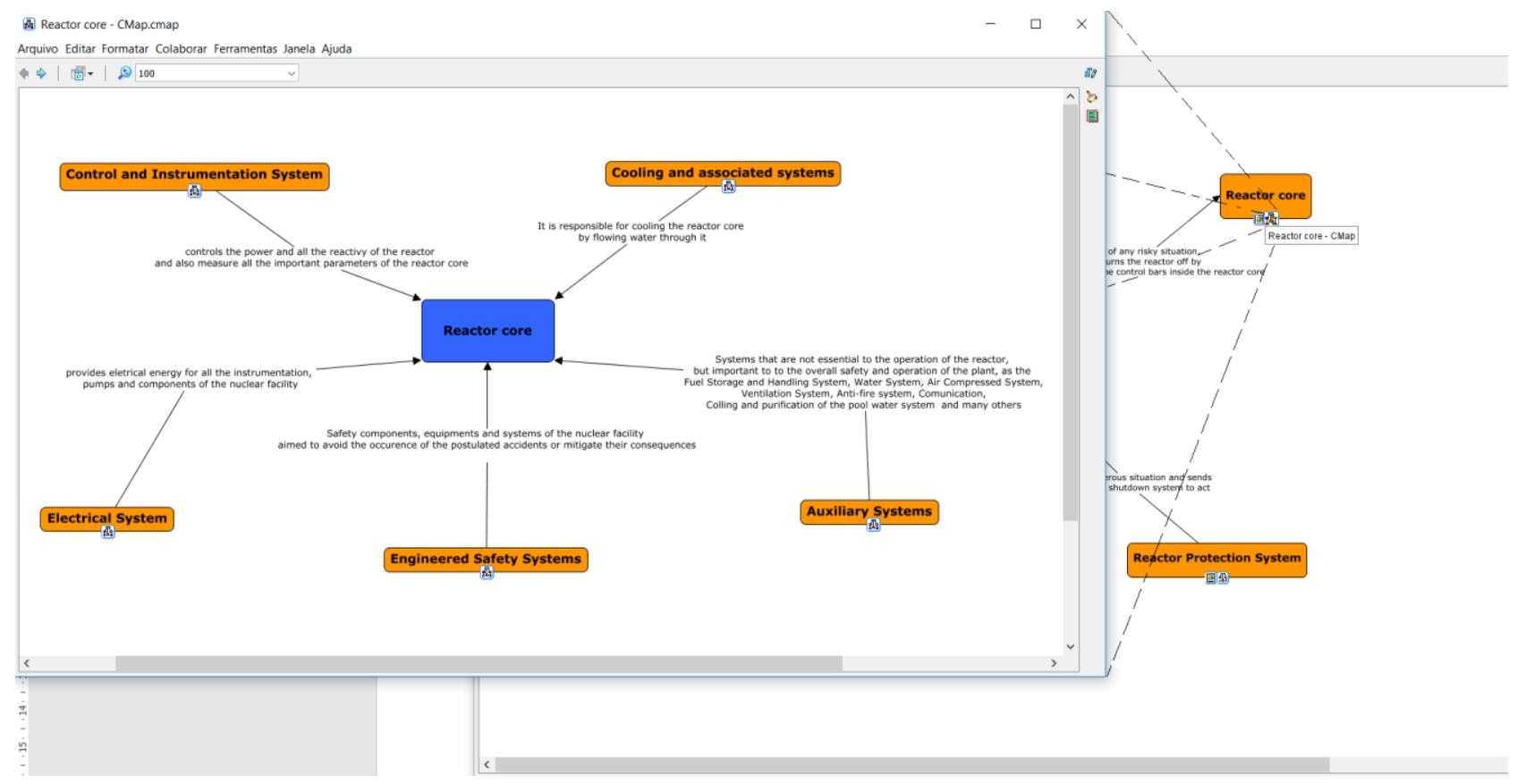

The knowledge base should provide information about the plant systems so that the user can understand how the system works and also get informed about which information is not yet defined, i.e., the pending issues, and which inconsistencies are found in the project documentation. The pending issues, and especially the inconsistencies, are elements that generate epistemic uncertainties and should be eliminated throughout the project.

Usually, there is not in the documentation related to typical large scale projects a document listing the pending issues (such as a list of the setpoints to be defined, for example) and the inconsistencies in the project documents. A document gathering this information could mitigate delays to the project and avoid the proliferation of epistemic uncertainties throughout the project's 
development. Therefore, in this knowledge base, it is proposed a document gathering all the pending issues, inconsistencies, and a description to each of the systems.

An example of this document, which is called "System Description", is shown in Figure 3. The user can view this document, which is displayed as a table, by clicking the visual block in the interconnection diagram where the system is included. To highlight the inconsistencies and pending issues, the yellow and red colors are used in the respective fields of the table.

Figure 3. Representation of the FSS description document popping up from a link located over the FSS visual block in the interconnection diagram.

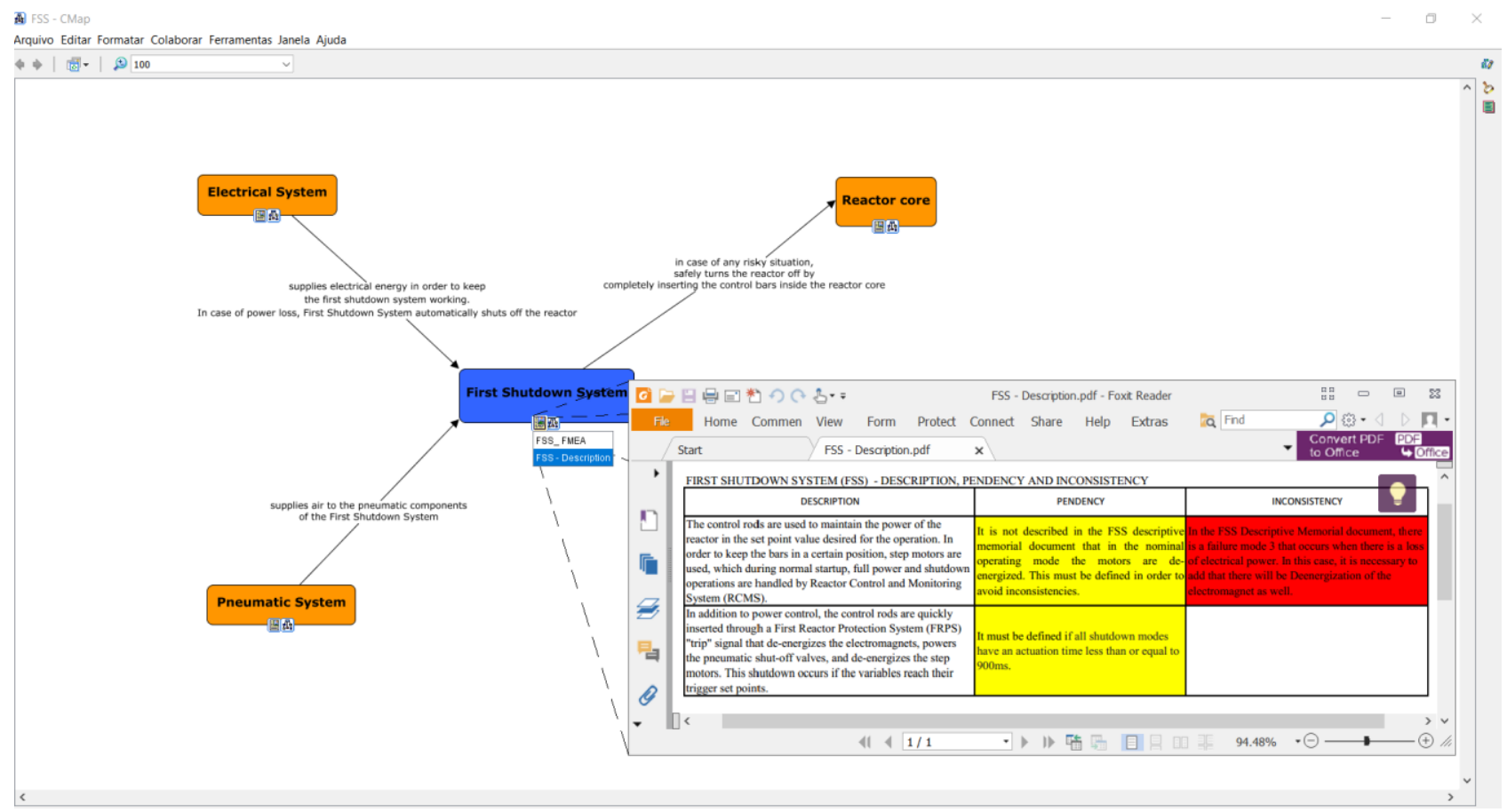

Any document considered important to a given system can be easily added to its visual block in its interconnection diagram by the user. As an example, Failure Modes and Effects Analysis (FMEA) can be attached to the knowledge base by the Safety Analyst who is working in the project, as exemplified in Figure 4, where a symbolic FMEA document of the FSS system pops up when the user clicks on its icon over the FSS visual block. 
The last level of the interconnection diagrams converges to the P\&I diagram of the described system, as represented in Figure 5 where it shows the FSS P\&I diagram popping up from them when the user clicks on the FSS visual block of its interconnection diagram.

Figure 4. Representation of the Failure Mode and Events Analysis (FMEA) document popping up from a link located in the FSS visual block of its interconnection diagram.

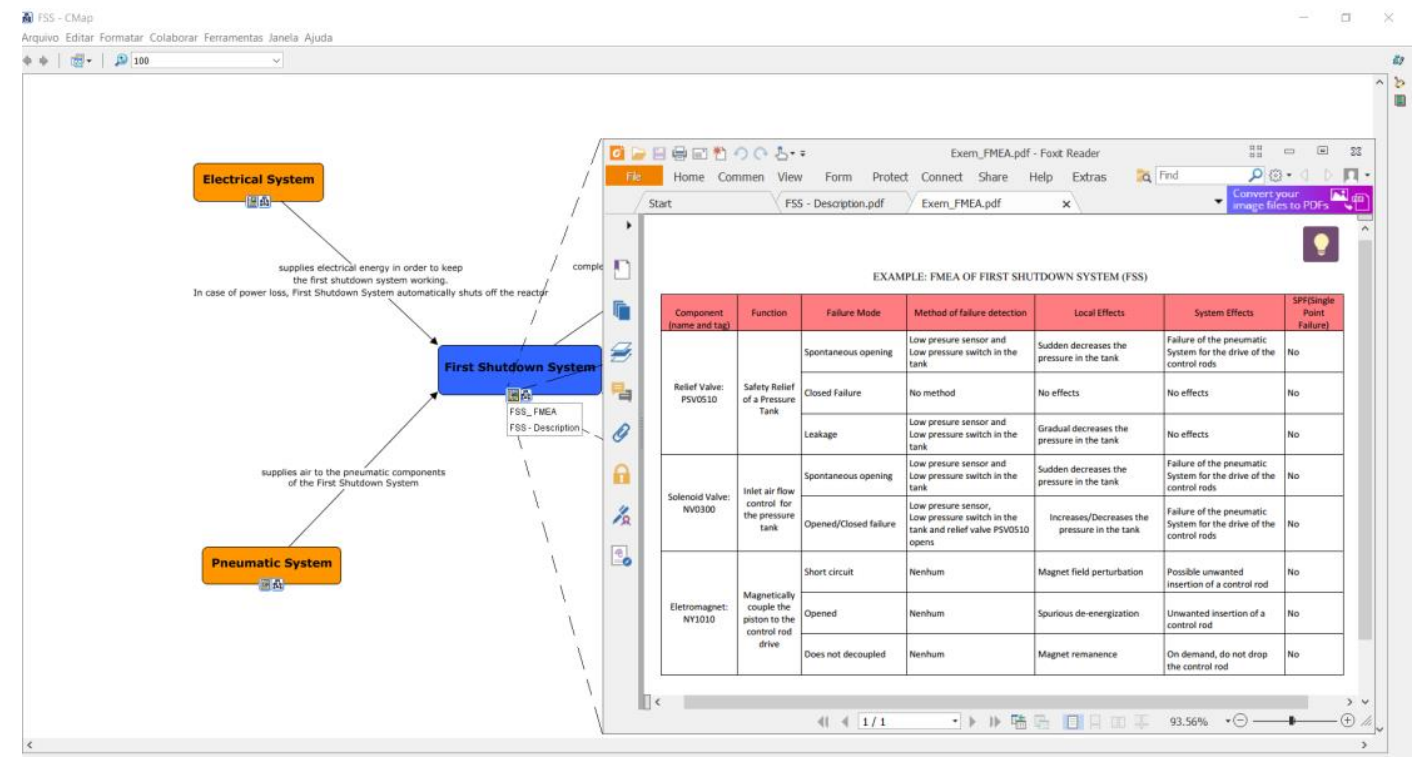

Figure 5. Representation of the FSS P\&I diagram popping up when the user clicks on the FSS visual block of its interconnection diagram.

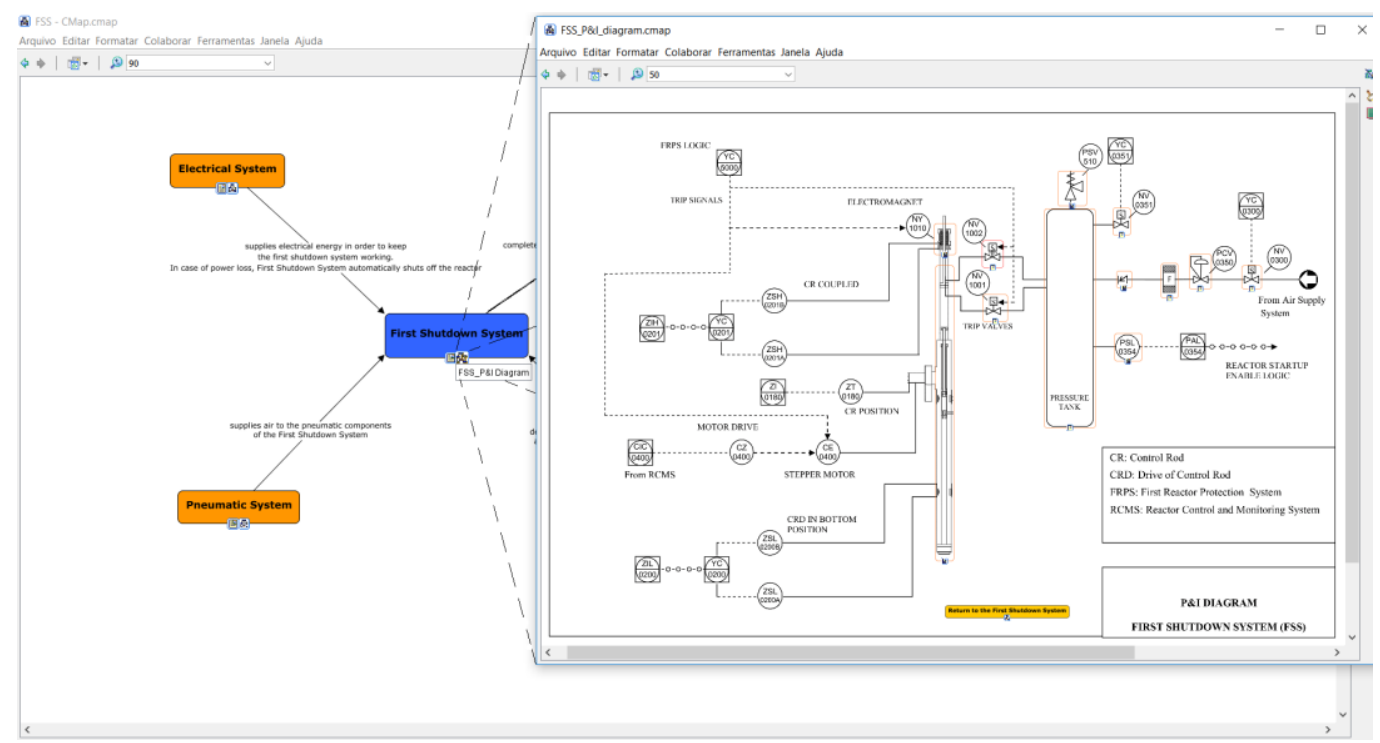


In the P\&I diagrams, over each component, there is a document presenting the information about the component, such as a description of how the component works, its reliability data, fabrication materials, failure modes, etc. This is not a permanent document, so it can always be updated by the users with the missing or correct details about the component. By the addition of this document, the user can have a broader knowledge about the component than just its reliability data (which is in most cases the only information given in reliability databanks, such as IAEA TECDOC- 930 or ANSI/IEEE STD 500 [7, 8]). Providing this document directly over the drawings of the components of the P\&I diagrams contributes to creating a holistic view about the influence of the component on the system it is applied and thus promoting a more accurate safety analysis of the system. Besides that, organizing and displaying the information by this method makes it fast, easy, and intuitive for the user to reach that given information. In Figure 6 an example of this document is shown with the datasheet of a pneumatic solenoid valve that is applied in the FSS system of the OPLRR. There is a description datasheet that opens when the user clicks over each component presented on the P\&I diagram, as represented in the figure. This description datasheet contains details about the component, such as its manufacture, model, main construction materials, common failures, maintenance, and some other details.

Figure 6. Example of the description datasheet with details about the component.

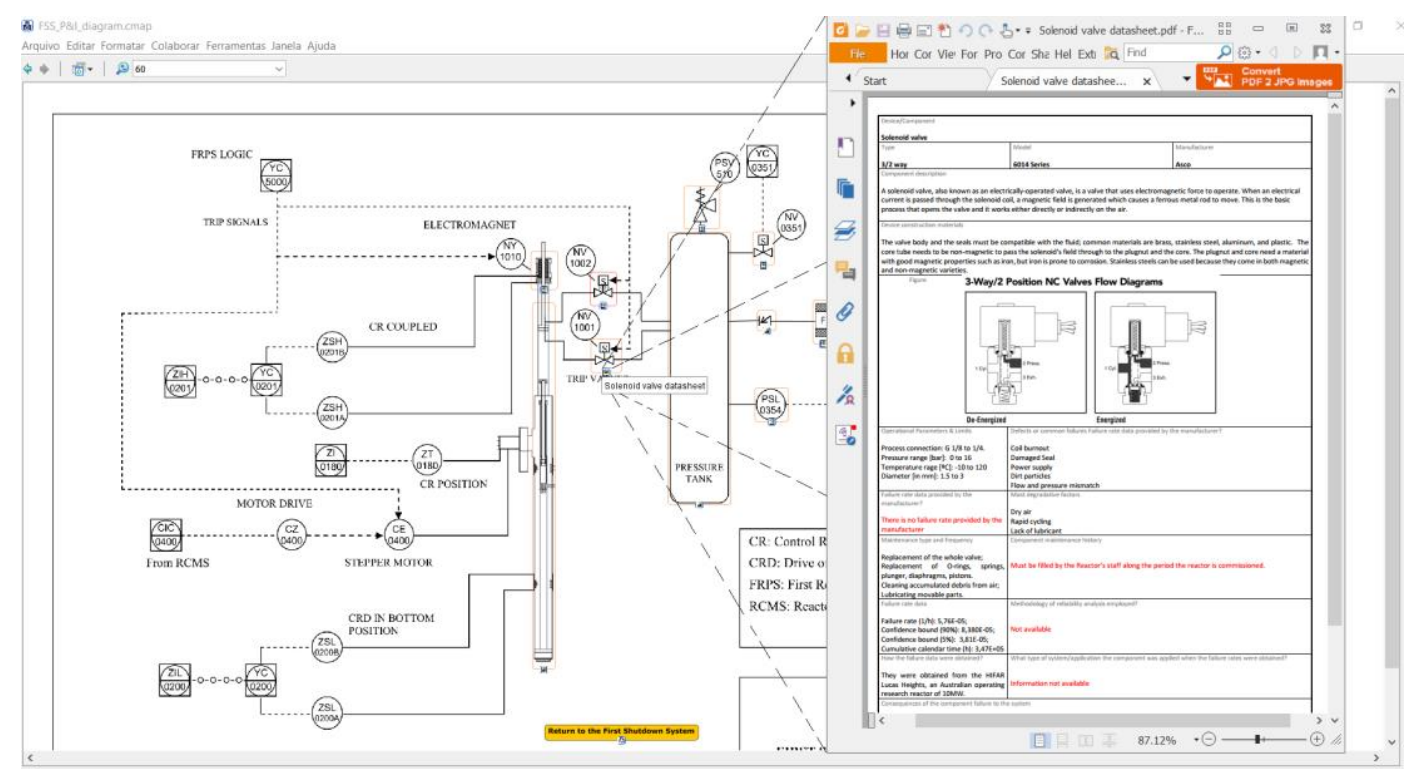


It is also provided another document dedicated to the reliability data of the component, as exemplified in Figure 7. A reliability datasheet of the component is available on each component presented at the P\&I Diagram of the knowledge-base. The user can visualize this document by clicking on the link over the component drawing, as represented in this figure.

Figure 7. Example of a reliability datasheet of the component.

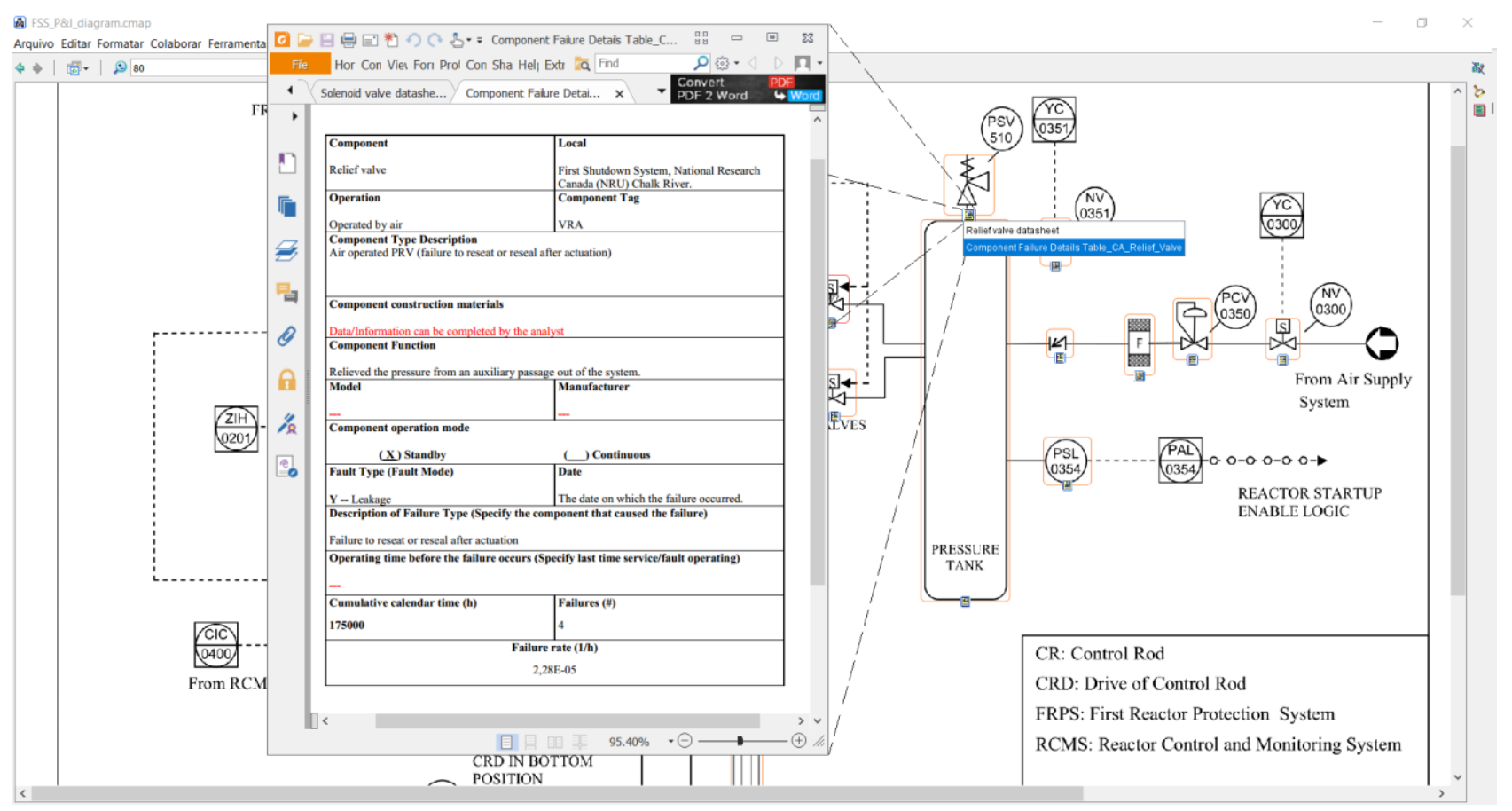

\section{DISCUSSION}

The graphical, hierarchical, and organized architecture of the proposed knowledge base allows the analyst or manager to quickly and easily obtain information regarding the systems, processes, equipment, and components of the project. Additionally, the proposal is that this knowledge base should be dynamic, i.e., it can be expanded and updated whenever it is considered necessary to include other information that is relevant to the project or the safety analysis.

The information contained in the System Description documents, Reliability, and Description Datasheets of the components comes from research and/or survey data on a particular system, 
equipment, or component. This process of research or selection of information is crucial to reduce the epistemic uncertainties, once it raises the issues and the inconsistencies of the project explains the interrelations between the systems and promote better comprehension of how the equipment works, its failures modes and consequences. All these and other important information, which are not originally contained in the project documents, contribute to reducing epistemic uncertainties arisen throughout the development of the project.

\section{CONCLUSION}

This paper presents a knowledge base in which information is organized and structured in a manner that contributes to reduce epistemic uncertainties and, thus, to collaborate in the processes of safety analysis and decision making. Besides being organized easily and intuitively, in a structure similar to that used in concept maps, the proposed knowledge base adds a set of information not listed in the project documentation or reliability databases, such as inconsistencies, ambiguities, and pendency's found at the project documents, as well as the contextualized descriptions of the equipment and components in the systems, which cooperates with the decision-making and security analysis processes and, at the same time, makes this knowledge base a novelty to the nuclear field. 


\section{ACKNOWLEDGMENT}

The authors thankfully acknowledge the financial support provided by FINEP- Financiadora de Estudos e Projetos and CNPq- Conselho Nacional de Desenvolvimento Científico e Tecnológico.

\section{REFERENCES}

[1] RAMASESH, R. V. and BROWNING, T. R. A conceptual framework for tackling knowable unknown unknowns in project management. Journal of Operations Management, Vol. 32, Issue 4, pp. 190-204 (2014).

[2] TSU, L. Tao Te Ching. Vintage Books, New York, sect. 71 (1972).

[3] KNIGHT, F.H. Risk, Uncertainty and profit. Houghton Mifflin Company, Boston and New York (1921).

[4] WALKER, T. W. Geographies of risk, uncertainty and ambiguity: A participatory action research project in catchment management. Ph.D. thesis for the degree of Doctor of Philosophy in Geography, University of Exeter (2016).

[5] KEUR, P. van der; HENRIKSEN, H. J.; REFSGAARD, J. C.; BRUGNACH, M.; PAHLWOSTL, C.; DEWULF, A.; BUITEVELD, H. Identification of Major Sources of Uncertainty in Current IWRM Practice. Water Resources Management, Vol. 22, Issue 11, pp 1677-1708 (2008).

[6] FLORIDA INSTITUTE FOR HUMAN \& MACHINE COGNITION (IHMC). Software Cmap Tools ${ }^{\circledR}$.

[7] INTERNATIONAL ATOMIC ENERGY AGENCY (IAEA), Generic Component reliability data for research reactor PSA, IAEA-TECDOC-930, IAEA, Vienna, Austria (1997).

[8] INSTITUTE OF ELECTRICAL AND ELECTRONICS ENGINEERING (IEEE). Recommended Practice for the Design of Reliable Industrial and Commercial Power Systems, ANSI IEEE STD 500. New York, USA (1984). 